\title{
Tuba-ovarian auto-amputation caused by ovarian teratoma in an adolescent girl
}

\author{
Ahmet Atıc1 ${ }^{1}$, Engin Yılmaz ${ }^{1}$, Ayşe Karaman¹, Sema Apaydın², Çağatay Evrim Afşarlar ${ }^{1}$ \\ Department of ${ }^{1}$ Pediatric Surgery and 2Pathology, Dr.Sami Ulus Maternity, Children's Health and Diseases Training \\ and Research Hospital, Ankara, Turkey. E-mail: ahmetim1501@hotmail.com \\ Received: 12th July 2016, Revised: 22nd July 2016, Accepted: 16th August 2016
}

SUMMARY: Atıcı A, Yılmaz E, Karaman A, Apaydın S, Afşarlar ÇE. Tubaovarian auto-amputation caused by ovarian teratoma in an adolescent girl. Turk J Pediatr 2017; 59: 90-92.

Ovarian auto-amputation is an extremely rare condition commonly encountered in the perinatal period. Spontaneous or secondary torsion of the ovary caused by an ovarian lesion may result in infarction and subsequent auto-amputation of the ovary. This paper demonstrates a case that underwent laparoscopic appendectomy with an incidental calcified auto-amputated right ovary.

A 16-year-old adolescent girl was admitted to our department with a history of one-day abdominal pain. Physical examination of the patient revealed abdominal tenderness and rigidity on right lower quadrant. Her white blood cell count was $11 \times 10^{3} / \mu \mathrm{L}$, and C-reactive protein was $69 \mathrm{mg} / \mathrm{L}$. The patient underwent a laparoscopic appendectomy with a provisional diagnosis of acute appendicitis, and further exploration revealed a $2 \times 2 \mathrm{~cm}$ white ovoid mass floating freely in the pelvis. The left ovary was clearly identified in its usual localization, but the right tuba was blindly ending without any fimbria or ovary. Postoperative course of the patient was uneventful, and she was discharged on postoperative day 2. The histopathological examination revealed a necrotic calcified ovarian teratoma.

Auto-amputated ovary is a rare occasion mostly encountered during perinatal period, and it may be unilateral or bilateral. An auto-amputated ovarian mass may rarely be a teratoma although the most common cause of autoamputation during perinatal and adolescent period is ovarian torsion due to an ovarian cyst.

Key words: adolescent, ovary, auto-amputation, teratoma.

Ovarian auto-amputation is an extremely rare condition frequently encountered in the perinatal period ${ }^{1}$. Giovanni Morgagni defined this state in a postmortem case for the first time in $1748^{2}$. This paper demonstrates a case that underwent laparoscopic appendectomy with an incidental calcified auto-amputated right ovary.

\section{Case Report}

A 16-year-old adolescent girl was admitted to our department with the history of oneday abdominal pain and anorexia. Her past medical history was not remarkable except for an episode of nonspecific abdominal pain that lasted for two days, five years before her admission. Physical examination of the patient revealed abdominal tenderness and rigidity on right lower quadrant. Her white blood cell count was $11 \times 10^{3} / \mu \mathrm{L}$, and C-reactive protein was $69 \mathrm{mg} / \mathrm{L}$. Abdominal $\mathrm{X}$-ray was not remarkable, and abdominal ultrasonography (US) was unable to show an inflamed appendix. Regarding the physical examination findings, surgical exploration was justified for a provisional diagnosis of acute appendicitis. The patient underwent a laparoscopic exploration. In addition to inflamed appendix, laparoscopic exploration demonstrated a white ovoid mass, which was freely floating in the deep pelvis without any attachment to viscera. The left ovary was clearly identified in its usual localization, but 
the right ovary was absent, and the right horn of uterus blindly ended $2-3 \mathrm{~cm}$ away, without any fimbria. The mass was removed from the abdominal cavity after completing a standard laparoscopic appendectomy. The mass was firm, calcified, and had a size of 2 (length) $\times 2$ (width) $\mathrm{cm}$ (Fig. 1). Postoperative course of the patient was uneventful. The histopathological examination of appendix was compatible with acute phlegmonous appendicitis, and histopathology of the ovoid mass revealed a necrotic calcified ovarian teratoma (Fig. 2).

\section{Discussion}

Auto-amputation caused by adnexal torsion is an uncommon condition, and the incidence has been found as $1 / 11,421^{2}$. A calcified autoamputated ovary wandering in the abdominal cavity is called as AWCO (An auto-amputated

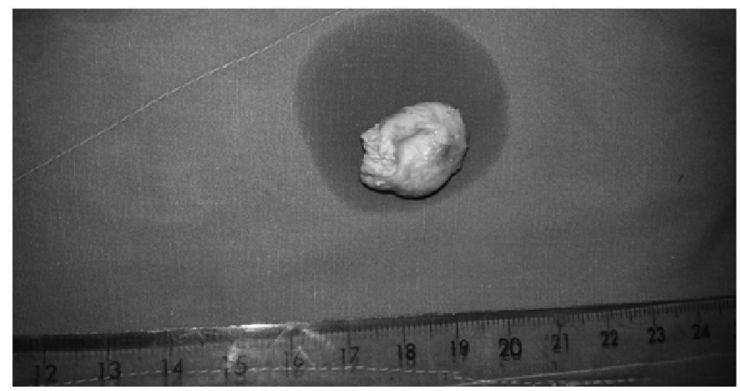

Fig.1. The macroscopic appearance of white ovoid mass removed from deep pelvis on the right side. wandering calcified ovary) in the pediatric population ${ }^{3}$. Occasionally, the remnant of auto-amputated ovary may completely be resorbed, and may not be visible ${ }^{2}$. However, calcification usually takes place, and prevents total resorption of the ovary ${ }^{3}$.

An auto-amputated ovary may be unilateral or bilateral. Left sided ovarian torsion is less common due to protective effect of sigmoid colon ${ }^{4}$. As in our case, right sided ovarian torsion is encountered more frequently ${ }^{3}$.

Devascularization and auto-amputation due to chronic adnexal torsion is the widely accepted theory for ovarian auto-amputation ${ }^{2}$. Then dystrophic calcification occurs, and autoamputated ovary floats freely in the abdominal cavity $^{3}$. The majority of the cases demonstrate ipsilateral blind ending fallopian tubes during laparoscopic exploration. The reported case was incidentally identified during a laparoscopic appendectomy procedure.

Pelvic calcifications in adolescent girls may also be associated with teratoma, chronic ovarian inflammation, appendicolith, urinary tract stones, calcified lymph nodes, Meckel's diverticulum, biliary stones, appendix epiploica torsion, or pelvic bone neoplasms ${ }^{2}$.

In the literature, it was indicated that patients with auto-amputated ovaries might exhibit chronic or intermittent pain, vomiting, and abdominal mass or might completely be

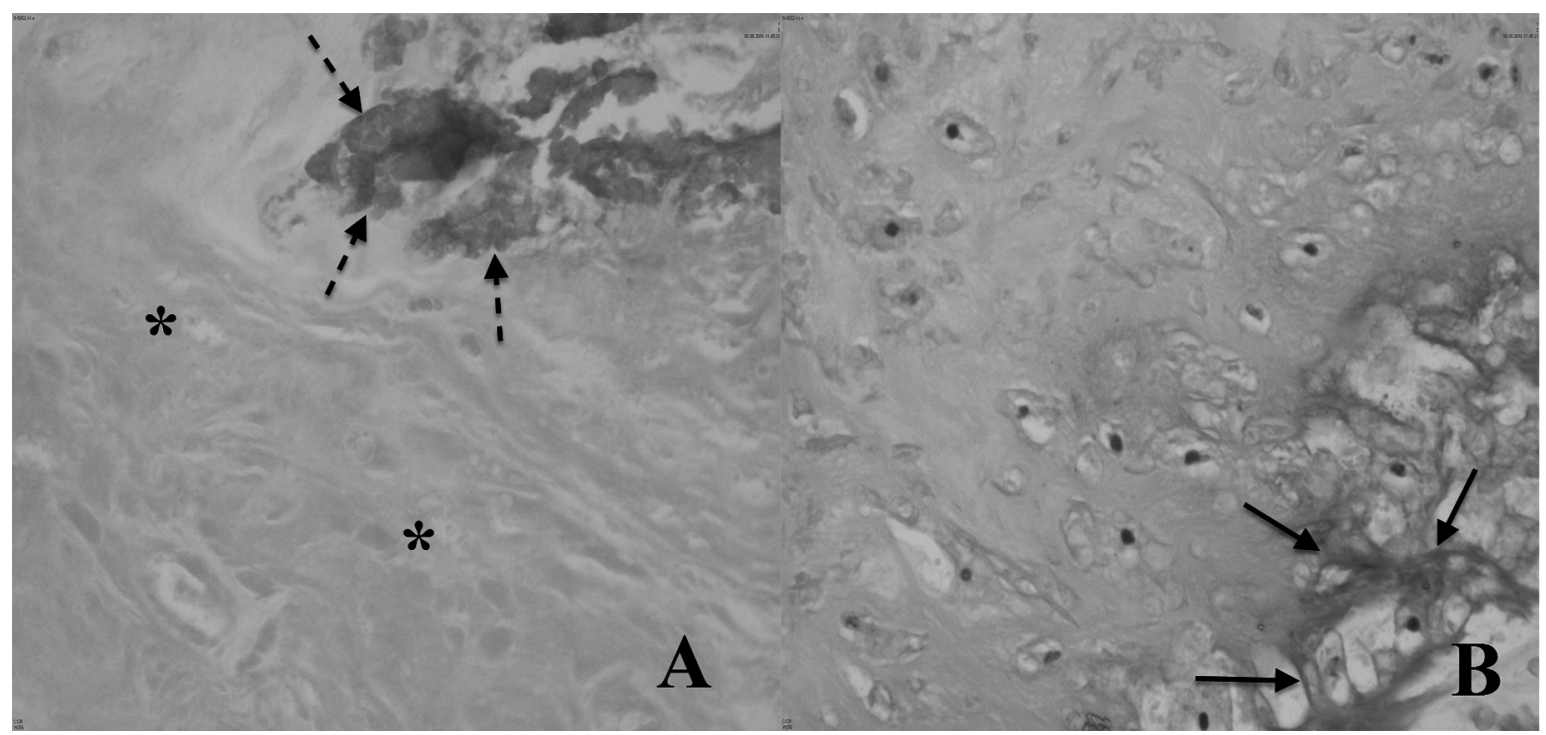

Fig. 2. Bone and cartilage tissue silhouette in the mass. (A) The more pink patchy areas represent bone (dashed arrows), and light pink areas demonstrate coagulation necrosis (asterisk). (B) The bluish areas represent cartilage (straight arrows). 
asymptomatic. As in our case, most of the patients are recognized incidentally during imaging studies or surgical interventions. Our patient did not have any significant medical history except for a nonspecific abdominal pain that lasted two days, five years before her admission. The patient did not exhibit any other pathological signs on plain abdominal X-ray or US studies other than acute appendicitis during preoperative evaluation. She did not have any prenatal US imaging or any other imaging studies until she was referred to our department.

Although a significant number of intrauterine, neonatal and adolescent cases with ovarian auto-amputation have been reported, most of the histopathological specimens evidently reveal calcified necrotic tissues ${ }^{4}$. Auto-amputation relevant to teratoma is an extremely rare occasion, as demonstrated in our case ${ }^{2}$ (Fig. 2).

Currently, widely available prenatal and postnatal imaging modalities have enabled diagnosis of numerous fetal and neonatal adnexal masses. Auto-amputation of ovaries due to ovarian cysts may be asymptomatic during antenatal period. A study that included 97 cases with auto-amputated ovaries revealed that $24.7 \%$ of the patients had ovarian cysts in prenatal history ${ }^{3}$. Of the auto-amputated ovaries, $50.5 \%$ were encountered under the age of 18 , and $13 \%$ were encountered during adolescent period as exhibited in our case, and most of those cases were determined during laparotomy or laparoscopy.
Parents usually wonder how the fertility will be affected when their children are diagnosed with an auto-amputated ovary. If the contralateral ovary is normal appearing, making relaxing explanations to the parents is suggested ${ }^{2}$.

Auto-amputated ovary is a rare occasion mostly encountered during perinatal period, and it may be unilateral or bilateral. As in our case, it is usually identified incidentally, and right side is the most frequent localization. An auto-amputated ovarian mass may rarely be a teratoma although the most common cause of auto-amputation during perinatal and adolescent period is ovarian torsion due to an ovarian cyst.

\section{REFERENCES}

1. Trotman GE, Zamora M, Gomez-Lobo V. Non-surgical management of the auto-amputated adnexa in the neonate: a report on two cases. J Pediatr Adolesc Gynecol 2014; 27: 107-110.

2. Focseneanu MA, Omurtag K, Ratts VS, Merritt DF. The auto-amputated adnexa: a review of findings in a pediatric population. J Pediatr Adolesc Gynecol 2013; 26: 305-313.

3. Mahajan PS, Ahamad N, Hussain SA. First report of MRI findings in a case of an autoamputated wandering calcified ovary. Int Med Case Rep J 2014; 7: 49-52.

4. Mellor A, Grover S. Auto-amputation of the ovary and fallopian tube in a child. Aust NZ J Obstet Gynaecol 2014; 54: 189-190.

5. Koga K, Hiroi H, Osuga Y, Nagai M, Yano T, Taketani Y. Autoamputated adnexa presents as a peritoneal loose body. Fertily and Sterility 2010; 93: 967-968. 